\title{
The Size, Symmetry, and Color Saturation of a Male Guppy's Ornaments Forecast His Resistance to Parasites
}

\author{
Jessica F. Stephenson, ${ }^{1,2,3, \star}$ Martin Stevens, ${ }^{4}$ Jolyon Troscianko, ${ }^{4}$ and Jukka Jokela ${ }^{2,5}$ \\ 1. Department of Biological Sciences, University of Pittsburgh, Pittsburgh, Pennsylvania 15260; 2. Department of Aquatic Ecology, Swiss \\ Federal Institute of Aquatic Science and Technology (EAWAG), Dübendorf 8600, Switzerland; 3. Center for Adaptation to a Changing \\ Environment (ACE), Eidgenössische Technische Hochschule (ETH) Zürich, Zurich 8092, Switzerland; 4. Centre for Ecology and Con- \\ servation, College of Life and Environmental Sciences, University of Exeter, Penryn Campus, Penryn, United Kingdom; 5. Institute for \\ Integrative Biology, ETH Zürich, Zurich 8092, Switzerland
}

Submitted February 26, 2020; Accepted June 24, 2020; Electronically published September 22, 2020

Online enhancements: supplemental PDF. Dryad data: https://doi.org/10.5061/dryad.547d7wm61.

\begin{abstract}
AвsтRAcт: Sexually selected ornaments range from highly dynamic traits to those that are fixed during development and relatively static throughout sexual maturity. Ornaments along this continuum differ in the information they provide about the qualities of potential mates, such as their parasite resistance. Dynamic ornaments enable real-time assessment of the bearer's condition: they can reflect an individual's current infection status, or they can reflect resistance to recent infections. Static ornaments, however, are not affected by recent infection but may instead indicate an individual's genetically determined resistance, even in the absence of infection. Given the typically aggregated distribution of parasites among hosts, infection is unlikely to affect the ornaments of the vast majority of individuals in a population: static ornaments may therefore be the more reliable indicators of parasite resistance. To test this hypothesis, we quantified the ornaments of male guppies (Poecilia reticulata) before experimentally infecting them with Gyrodactylus turnbulli. Males with more left-right symmetrical black coloration and those with larger areas of orange coloration, both static ornaments, were more resistant. However, males with more saturated orange coloration, a dynamic ornament, were less resistant. Female guppies often prefer symmetrical males with larger orange ornaments, suggesting that parasite-mediated natural and sexual selection act in concert on these traits.
\end{abstract}

Keywords: carotenoid coloration, Hamilton-Zuk hypothesis, sexual selection, parasite resistance, fluctuating asymmetry, static and dynamic traits.

\section{Introduction}

Individuals use multiple ornaments during their assessment of potential mates. These range from highly dy-

* Corresponding author; email: jess.stephenson@pitt.edu. ORCIDs: Stephenson, https://orcid.org/0000-0001-8939-5149; Stevens, https://orcid.org/0000-0001-7768-3426; Troscianko, https://orcid.org/0000 -0001-9071-2594; Jokela, https://orcid.org/0000-0002-1731-727X.

Am. Nat. 2020. Vol. 196, pp. 000-000. (C) 2020 by The University of Chicago. 0003-0147/2020/19605-59805\$15.00. All rights reserved.

DOI: $10.1086 / 711033$ namic traits that change on a second-to-second basis, enabling real-time assessment of the bearer's condition, to traits fixed during development that reflect the bearer's genotype quality and early-life experiences but are relatively static during sexual maturity (Hill et al. 1999). Traits along this continuum provide very different information about a potential mate's (hereafter assumed to be male) quality, such as how he interacts with parasites. Broadly, a choosing individual (hereafter assumed to be female) may consider parasites in two ways: (i) whether the male is currently infected and (ii) how resistant he is to infection. Dynamic and static ornaments are differentially informative on each of these points. For example, dynamic ornaments change rapidly in response to changes in male condition (Wingfield et al. 1990; Folstad and Karter 1992; Hill et al. 1999) and can therefore provide information about a male's current parasite infection: males with the most exaggerated dynamic ornaments in a population are unlikely to be infected and, importantly for choosing females, not contagious (Loehle 1995; Able 1996). Static ornaments, by contrast, are not useful indicators of present infection. By comparing the dynamic ornaments of infected and uninfected males in a population, females can reliably choose uninfected males and thus avoid becoming infected during copulation (Loehle 1995; Able 1996). This contagion indicator hypothesis is well supported by empirical work across taxa (e.g., Borgia 1986; Milinski and Bakker 1990; Siva-Jothy 2000; Meadows et al. 2011).

In addition to choosing males on the basis of their current infection status, females may also use male ornaments to choose relatively resistant mates. Despite the potential usefulness of static traits in signaling parasite resistance, previous explorations of this idea have focused exclusively on dynamic ornaments. Most notably, the Hamilton-Zuk and immunocompetence handicap hypotheses both posit that among the currently or recently infected males in a 
population, only the most resistant should be able to maintain the most exaggerated dynamic ornaments (Hamilton and Zuk 1982; Folstad and Karter 1992). Hamilton and Zuk further suggested that through mating with these resistant males, females have offspring with genetically determined resistance to local parasite strains and, because of the coadaptational cycles between hosts and parasites, heritable genetic variation in dynamic ornaments is maintained (Hamilton and Zuk 1982). Since it was proposed, the predictions made by the Hamilton-Zuk hypothesis have been tested many times across animal taxa, but the evidence remains mixed (reviewed by Hamilton and Poulin 1997; Møller et al. 1999; Weaver et al. 2018).

The role of static ornaments in signaling parasite resistance has not been explicitly considered, but given the ecology of most host-parasite interactions, they may provide more reliable information about a male's parasite resistance than his dynamic traits. Because by definition they do not reflect recent changes in male condition, static ornaments are less sensitive to stochastic factors and may more closely reflect a male's genetic quality (Hill et al. 1999; Møller et al. 1999). In particular, static ornaments are not vulnerable to the "noise" introduced by parasite distribution in natural conditions: given the typically aggregated distribution of parasites among their hosts (Shaw and Dobson 1995), relatively few males in any population will be infected and, among these, infection loads may rarely reach levels that affect dynamic male ornaments (Poulin and Vickery 1993). Static ornaments may also be less vulnerable to cheating: dynamic ornaments require less long-term investment (Wingfield et al. 1990; Folstad and Karter 1992; Hill et al. 1999).

There are at least two elements of static male ornaments that are promising candidates for signaling parasite resistance. First, deviation from perfect symmetry around a mean of zero (i.e., "fluctuating asymmetry") arises during the development of morphological structures and tends to be highest in secondary sexual traits (Palmer and Strobeck 1986; Sheridan and Pomiankowski 1997b; Møller et al. 1999). Fluctuating asymmetry in secondary sexual traits may be dynamic-for example, if these traits are renewed between breeding seasons (e.g., deer antlers) - but is static in traits that develop once, such as those examined here. Fluctuating asymmetry is hypothesized to correlate negatively with fitness, but empirical evidence for this relationship remains equivocal (Lens et al. 2002). However, resistance to parasites correlates negatively with fluctuating asymmetry in other systems (e.g., in mosquitoes; Agnew and Koella 1997), and symmetry correlates with ability to withstand stress in fishes (Allenbach et al. 1999).

Second, carotenoid coloration is a common feature of male ornamentation across taxa and has both static and dynamic elements. Commonly, the area of a male's carotenoid coloration, such as beak size in blackbirds or spot size in guppies, is a static trait. Saturation (i.e., the richness of a color compared with the achromatic point: red is more saturated than pink), on the other hand, can be highly dynamic: both blackbird beak (Faivre et al. 2003) and guppy spot saturation (Houde 1997) can change on a scale of minutes. The role of carotenoid coloration in signaling parasite resistance has received a vast amount of research attention because vertebrates must assimilate carotenoids from their diets (Goodwin 1986) and, in addition to pigmentation, carotenoids boost immune function (Lozano 1994; Grether et al. 2004a; Kolluru et al. 2006). Consequently, if the area and saturation of a male's carotenoid coloration are positively correlated with his ability to assimilate carotenoids, they may reliably indicate his ability to defend himself against parasites (Lozano 1994). Indeed, across taxa females often prefer males with larger, more saturated carotenoid coloration (e.g., Kodric-Brown and Brown 1984; Andersson 1994; Blount et al. 2003).

We here quantify multiple static and dynamic ornaments in laboratory-bred, parasite-naive virgin males before assessing their resistance to an experimental infection of the most prevalent macroparasite in their habitat. While most previous studies of the correlation between male ornamentation and parasite resistance have quantified male ornamentation after parasite exposure, a handful of previous studies quantifying single ornaments before parasite exposure indicates they can indeed forecast parasite resistance. Dawson and Bortolotti (2006) observed that male kestrels with more saturated carotenoid coloration at the start of the breeding season are more likely to resist parasites later in the breeding season. In experimental infections, redder male house finches are able to clear $\mathrm{Myco}$ plasma gallicepticum infection faster than yellower males (Hill and Farmer 2005), male greenfinches with brighter tail plumage clear virus infection faster than those with duller tails (Lindström and Lundström 2000), and wolf spiders with more symmetrical leg tufts develop lower bacterial infection loads (Gilbert and Uetz 2016). Building on these results, we tested whether the left-right symmetry and size of a male guppy's (Poecilia reticulata) ornaments (both static traits) or their color saturation (a dynamic trait) more reliably forecast his resistance to subsequent experimental infection with a highly prevalent macroparasite, Gyrodactylus turnbulli. We found that males with more attractive static ornaments were more resistant to infection, whereas those with more attractive dynamic ornaments were less resistant. Because we quantified color saturation before infection, this result may indicate that males trade off dynamic ornamentation and parasite resistance. In support of this idea, we found that the dynamic ornament, but not the static, was significantly negatively correlated with 
male body condition. By demonstrating that male ornaments forecast resistance to parasites but that the direction may depend on the ornaments' dynamism, these results broaden our understanding of the role of parasites in sexual selection.

\section{Material and Methods}

\section{System}

The guppy-Gyrodactylus turnbulli system is well suited to testing the relative reliability of dynamic and static male ornaments in forecasting parasite resistance. The parasite imposes fitness costs and is the most prevalent macroparasite in natural populations (Houde and Torio 1992; van Oosterhout et al. 2007; Stephenson et al. 2015a, 2015b, 2016). Our understanding of male coloration, as with other aspects of guppy ecology and evolution, comes from multiple studies conducted across different natural populations. Whether and how populations may vary is often unclear, and this uncertainty is an important caveat of the following summary. Male guppy coloration is highly polymorphic and comprises up to 10 discrete elements (Endler 1978; Endler 1980; Houde and Endler 1990): no two males have identical color patterns (Houde 1997). Male coloration is fixed during development and is fairly static through sexual maturity (but see Evans et al. 2002), so fluctuating asymmetry in color pattern elements can be considered a static trait in this system. The area and saturation of orange/yellow spots (hereafter, "orange") are important components of male ornaments (Houde 1997; Kemp et al. 2018) and arise from carotenoid (from dietary sources) and pteridine (nondietary) pigments (Grether et al. 2005). The area of orange pigmentation is under genetic control (Hughes et al. 2005), is fixed during development (but see Evans et al. 2002), does not appear to be strongly influenced by environmental factors (Kodric-Brown 1989; Grether 2000), and thus as a relatively static trait should be reflective of a male's genetic quality. By contrast, orange saturation is dynamic and condition dependent and thus may indicate a male's ability to thrive in the present environmental conditions (Endler 1980, 1983; Kodric-Brown 1989). Males fed a carotenoid-rich diet have more saturated orange spots (Kodric-Brown 1989; Grether 2000; Karino and Hajima 2004) and an increased acquired immune response, suggesting a potential trade-off between allocating carotenoids to sexual ornamentation or immune function (Folstad and Karter 1992; Grether et al. 2004a). Testing how these three components of male ornamentation (fluctuating asymmetry, orange area, and saturation) may correlate with a male's parasite resistance therefore helps to elucidate the relative importance of genetic quality and overall condition to this trait. Furthermore, they are important in mate choice in this system: female guppies from many populations prefer males with overall more symmetrical (between the two body sides) color patterns and larger areas of more saturated carotenoid coloration (Kodric-Brown 1989; Houde and Torio 1992; Sheridan and Pomiankowski 1997a; Karino et al. 2010).

\section{Host Origin and Maintenance}

We used first-generation laboratory-bred descendants of wild guppies from a high-predation population located at an undeveloped rural site on the Caura River, Trinidad (Universal Transverse Mercator: 20 P 679527.7 m E, $1180376.4 \mathrm{~m} \mathrm{~N}$, based on WGS84 datum; elevation, $112 \mathrm{~m})$. In this population, males with larger areas of orange pigmentation are preferred by females during both pre- and postcopulatory mate choice (Evans et al. 2003, 2004; Devigili et al. 2015). While it is clear that guppy populations differ in the extent to which orange pigmentation is composed of dietary carotenoids (which are known to have an immune function in guppies; Grether et al. 2004a; Kolluru et al. 2006) and nondietary pteridine pigments (which do not have an immune function; Grether et al. 2005), the relative importance of these pigment types to the orange pigmentation of the males in this population remains untested. However, the orange spots of males from all populations that have been tested do comprise at least some carotenoids: we are almost certain that carotenoids contribute to the orange pigmentation of males in our focal population - and perhaps substantially, given the relatively open canopy (thus high algal growth and carotenoid availability; Grether et al. 2001).

Wild guppies $(n \sim 600)$ were transported to Cardiff University in June 2012 (Centre for Environment Fisheries and Aquaculture Science Aquaculture Production Business authorization CW054-D-187A), where they were prophylactically treated for infection using nitrofurazone (Binox; Jungle Laboratories, Cibolo, Texas). This site was found to be free of Gyrodactylus spp. in a previous survey (Stephenson et al. 2015b), and we failed to find any Gyrodactylus spp. during examination of a subset of 80 of the collected wild fish. Fish were housed in 70-L mixed-sex stock tanks on a $12 \mathrm{~L}: 12 \mathrm{D}$ photoperiod (overhead fluorescent lighting) at $24^{\circ} \pm 1^{\circ} \mathrm{C}$ and fed daily with Aquarian tropical fish flakes supplemented with Artemia and bloodworms. Fry were removed from the breeding tanks soon after birth, confirmed free of ectoparasitic infection under a microscope, reared in one of four juvenile tanks until sex determination was possible ( $\sim-8$ weeks; males were identified by the initiation of the modification of the anal fin, females by dark pigmentation at the base of the anal fin), and then reared in sex-specific tanks for at least a further month before use. All males used in this experiment were therefore sexually mature virgins. 
Our use of first-generation laboratory-bred descendants of wild-caught fish means that we did not control the environment experienced by the parents of our experimental fish. Although unlikely based on our observations of our collected fish and their natural population, it is possible that some parents of our experimental fish had been exposed to parasites, and at least the mothers additionally experienced capture and transport to the laboratory. While there has been no explicit test of how parasite exposure, immune activation, or stress in guppy parents affects the ornamentation or parasite resistance of their offspring, Grether et al. (2008) found that maternal carotenoid intake did not affect male offspring coloration across fish from four Trinidadian populations. It is difficult to envisage how parental environment might drive the correlations we observed between male ornamentation and parasite resistance, but this could usefully be tested in future experiments.

\section{Color Pattern Data Collection}

Males $(n=27)$ were at least 2 months of age at the time of the experiment to ensure the full development of their color patterns (Houde 1997; van Oosterhout et al. 2003). Males were sedated using $120 \mathrm{mg} \mathrm{L}^{-1}$ MS222 for $60 \mathrm{~s}$ and transferred to a shallow petri dish on a uniform gray background. Anaesthetic solution was made in a large batch, and fresh solution was used for each individual. Fish were gently manipulated so the left and right sides could be photographed (Nikon D7000 fitted with an AF-S DX Micro Nikkor 85-mm f/3.5G lens), with a shutter speed of $1 / 250$, an aperture of $F=16$, and ISO 100 . The fish were illuminated indirectly with two remote flashes (Nikon R1 close-up speedlight). To test for the repeatability of measurements made of the same side of the same fish across different images, we took duplicate images of a subset of male sides.

All images were analyzed using the Multispectral Imaging plug-in for ImageJ (Schneider et al. 2012; Troscianko and Stevens 2015), which generates images that are linear with respect to radiance from digital camera RAW files and can be used to make reliable, quantitative measurements of animal coloration (Stevens et al. 2007; Troscianko and Stevens 2015). A single observer outlined all colored spots on each image as well as the body area of the fish (excluding all fins). We used the gray background (for which we quantified reflectance values) to standardize the images and then used the Multispectral Imaging plug-in's "batch multispectral image analysis" tool to calculate the area and RGB (red, green, blue) level of each spot on each fish side as well as fish body area. From these, we calculated spot color saturation as the distance of the RGB color measurement from the center of the trichromatic color space
(Stevens et al. 2009). We used these data to calculate the total saturation and area of carotenoid (orange) and melanin-based (black) coloration on each fish side (mean saturation of all spots on the left side relative to body area, plus mean saturation of all spots on the right side relative to body area) and the difference in black and orange area between the left and right sides of individual fish. We exclusively limit our study to the orange and black ornaments because they were by far the most common in our population (this is not universal across populations; e.g., Kemp et al. 2018) and have been the focus of many studies of both sexual selection and parasitism in guppies (Houde 1997; Houde and Torio 1992; Martin and Johnsen 2007; Gotanda et al. 2013).

Although structural color is an important component of male ornamentation in this system in general (Sheridan and Pomiankowski 1997a; Kemp et al. 2018) and in our focal population (Devigili et al. 2015), we did not include it in this study because in guppies it is often iridescent and therefore lacks clear boundaries, is very dependent on viewing and lighting angle, and is thus incredibly challenging to quantify robustly from single images (Sheridan and Pomiankowski 1997a; Meadows et al. 2011). We also did not quantify ultraviolet reflection from either the structural component or the pigment component of male ornaments, both of which are important to female mate choice in our focal population (White et al. 2003). That we were unable to test for correlations between parasite resistance and these additional aspects of male ornamentation does not change the conclusions we draw from the data we present here.

\section{Parasite Origin, Maintenance, and Experimental Infection Protocol}

We infected the experimental males after they had been photographed. The G. turnbulli strain we used, Gt3, was founded by a single parasite from an ornamental guppy in 1997 and has since been maintained on an inbred ornamental guppy stock ("culture fish"). To infect experimental males, culture fish were killed using an overdose of tricaine methanesulfonate (MS222; Pharmaq, Sandleheath, United Kingdom). We anesthetized the experimental males with $0.02 \%$ MS222 and recorded their standard length and weight before infecting them. Under a dissecting microscope, the tails of the culture and experimental fish were placed in close proximity until two individual parasites had transmitted. The experimental males were revived, placed in individual 1-L tanks, and maintained as described above. We monitored the trajectory of the infection on the males by anesthetizing each (0.02\% MS222) and counting the number of G. turnbulli every other day throughout 9 days of infection. While it is unknown how repeated exposure to MS222 may affect 
parasite growth rates, all fish (and therefore parasites) were exposed to the anaesthetic in the same way, so any anaesthetic-driven effects cannot explain our results.

Nine days of infection is sufficient to see differences between individual hosts in their ability to limit parasite establishment and population growth (see fig. S1, available online, and Stephenson et al. 2018). Typically in this system, parasite-induced host mortality increases dramatically at around day 10 of infection (Dargent et al. 2013). Males are less tolerant of Gyrodactylus infection than females (van Oosterhout et al. 2007; Stephenson et al. 2015a, 2016), and the parasite's population growth rate up to day 10 is a more important predictor of their survival than other parameters calculated from a longer infection trajectory in the laboratory (J. F. Stephenson, unpublished data). Additionally, field-based work has indicated that as infection intensities increase by a single worm, male survival decreases by $19 \%$ (van Oosterhout et al. 2007). An infection of 9 days is therefore sufficient to observe differences in male parasite resistance that are relevant to their survival under natural conditions.

We quantified resistance as the "infection integral," or the area under the curve of infection load over the 9 days of infection. This metric summarizes the intensity of an individual's infection over time, and because it uses several individual observations it is much less vulnerable to errors resulting from screening frequency than other common metrics, such as day of maximum parasite load, the maximum number of parasites, and instantaneous rates. As such, it has been used to great effect in this system (Stephenson et al. 2017, 2018; Phillips et al. 2018) and others (Adelman et al. 2013).

\section{Preliminary Analyses: Repeatability, Measurement Error, and Asymmetry}

All analyses were conducted in $\mathrm{R}$ statistical software ( $\mathrm{R}$ Core Team 2018; see the supplemental PDF, available online, for more details, script, and output), ${ }^{1}$ and all data underlying our results have been deposited in the Dryad Digital Repository (https://doi.org/10.5061/dryad.547d7 wm61; Stephenson et al. 2020). To test for the contribution of measurement and photographic error to our estimates, measurements were taken from each of two photographs of a subset of male sides. We used the rptR package (Stoffel et al. 2017) to test for the repeatability of measurements made across these replicate images. We used mixed model analysis of variance (ANOVA) to assess the relative contribution of measurement error to

1. Code that appears in The American Naturalist is provided as a convenience to readers. It has not necessarily been tested as part of peer review. our estimate of (signed) left-right asymmetry in area, following Palmer and Strobeck (1986). This ANOVA also provided a test for directional asymmetry in ornament area between the left and right sides of the fish (e.g., the left side having larger orange areas consistently across fish; Swaddle et al. 1994). To test for antisymmetry we used $t$-tests and Kolmogorov-Smirnov tests to confirm that the absolute differences in black and orange area between the left and right sides of the fish were centered on zero and normally distributed (Swaddle et al. 1994).

Finally, we tested for possible correlations between asymmetry in ornament area (i.e., the absolute difference between the left and right body sides) and fish body size or the size of the ornament (Swaddle et al. 1994). We assessed the suitability of a number of common indices of fluctuating asymmetry in minimizing these correlations: the raw difference (the absolute difference in color area in square millimeters between left and right sides), the relative difference (the raw difference divided by the mean size of the trait for that individual), the percentage difference (the raw difference divided by the body area of the individual), and the traditional difference (the raw difference divided by the larger of the trait sizes between the left and right sides). We also included a fifth index: the absolute difference need not be linearly associated with the size of the trait. We therefore ran models with the quadratic and cubic terms to test for the presence of such a relationship and used the residuals of these models as our index of fluctuating asymmetry. For orange area, the index with the lowest correlation with the size of the fish and the size of the ornament was the residuals of a model including the cubic and quadratic terms. For black, the best index was the raw difference between the left and right body sides.

\section{Data Analysis}

Our image analysis produced several ornament variables that could potentially predict parasite resistance. There were, predictably, some strong correlations between these variables, and we chose between them on the basis of our a priori hypotheses and to minimize the correlations among our final model variables. We chose to include area variables rather than spot count variables (which were highly correlated) and to include saturation variables rather than brightness variables. Where necessary, we chose individual color variables over summed color variables. We excluded black saturation because it was strongly negatively correlated with total black area (as well as having low repeatability; see below): the larger the area of black a fish had, the less saturated it was. Among our final model variables, the maximum correlation coefficient was 0.39 (see the supplemental PDF for more details and plots). 
To test whether male ornaments could significantly predict male guppy resistance to infection with G. turnbulli (quantified using the infection integral: the area under the curve of infection load through time), we used a generalized linear model (gamma error distribution and log link function). In the model, we included the following ornament variables as fixed effects: left-right symmetry of orange and black coloration, area of orange and black coloration, and orange saturation. We additionally included fish standard length and preinfection body condition (scaled mass index; Peig and Green 2009) to control for possible effects of fish size and condition on parasite population growth. We present the results of this full model, following Forstmeier and Schielzeth (2010).

As detailed below, the results indicated that males with more saturated orange ornaments were less resistant to $G$. turnbulli infection, suggesting that males may trade off the quality of these ornaments with parasite resistance. To further investigate this idea, we ran a post hoc analysis to test whether orange saturation, orange area, and black fluctuating asymmetry (i.e., the ornaments that significantly forecast parasite resistance; see below) were correlated with male body condition. We used each ornament variable as the response variable in three separate Gaussian linear models and included body condition and length as fixed effects. Our prediction was that only the dynamic ornament, orange saturation, should be correlated with body condition: unlike static ornaments, dynamic ornaments could be traded off with other resource-dependent traits as part of the individual's optimization strategy.

\section{Results}

\section{Preliminary Analyses}

The repeatability of the area measurements was high and significant for both black and orange coloration (both $R>$ $0.73, P<.0003)$. Repeatability of orange saturation was high $(R=0.70, P=.0017)$, but repeatability of black saturation was relatively low, although it was significant $(R=$ $0.52, P=.02$ ), possibly because of the known effects of anaesthetic on melanophores (Grether et al. 2004b) and the necessary time delay between replicate photographic images. This low repeatability, combined with its strong correlation with black area, meant that we removed black saturation from further analyses.

We found no evidence that guppy color patterns show directional asymmetry or antisymmetry. The side of the fish did not explain a significant amount of the variation in the area of orange or black (both $P>.3$ ), indicating no directional asymmetry. While left-right differences were centered on zero for both black and orange area, only the black area differences conformed to the normal distribu- tion. Left-right differences in orange deviated slightly but significantly $(D=0.265, P=.037)$ from normality, but there was no evidence for the broad peak or bimodality indicative of antisymmetry (see the supplemental PDF for plots and more details). Hereafter, therefore, we refer to the left-right differences in male color patterns as fluctuating asymmetry. Fluctuating asymmetry made a significantly larger contribution to the measured left-right differences than measurement error for both orange $\left(F_{14,28}=11.24\right.$, $P<.0001)$ and black $\left(F_{14,28}=8.29, P<.0001\right)$.

\section{Do Male Ornaments Predict Resistance to a Subsequent Infection?}

Using the infection integral as the response variable, we found that males with more symmetrical black coloration and larger areas of orange were more resistant to subsequent infection with Gyrodactylus turnbulli (i.e., had lower infection integrals), but larger males and those with more saturated orange coloration were less so (table 1; fig. 1). These results remained qualitatively unchanged after the removal of one individual that developed an outlying infection integral of 447 (table 1; see the supplemental PDF for more details).

\section{Are These Predictive Male Ornaments Correlated with Body Condition?}

Our post hoc analysis revealed that, as predicted, only dynamic orange saturation was correlated with body condition $\left(F_{1,24}=5.18, P=.032\right.$; both static traits $\left.P>.6\right)$. This correlation was negative and thus suggestive of a trade-off: males with the lowest body condition had the most saturated orange spots (fig. 2).

\section{Discussion}

Males with more symmetrical black ornaments and larger areas of orange, both static traits, were more resistant to the parasite, whereas larger males and those with more saturated orange coloration, a dynamic trait, were less resistant. We highlight that (i) male coloration in the absence of infection can be used as a reliable indicator of parasite resistance and (ii) static ornaments were positively correlated with parasite resistance, whereas (iii) the dynamic ornament was negatively correlated with resistance and, unlike the static ornaments, was also negatively correlated with male body condition. We conclude by describing the potential implications of these patterns for parasitism and selection in natural populations. 
Table 1: Generalized linear model evaluating whether the area, saturation, and symmetry of male color patterns forecast resistance to experimental infection with Gyrodactylus turnbulli

\begin{tabular}{lcccrc}
\hline Parameter & Standardized coefficient & Partial $R^{2}$ & Estimate (SE) & $\chi^{2}(\mathrm{df})$ & $P$ (without outlier) \\
\hline Condition & .176 & .038 & $.02(.01)$ & $1.76(1)$ & $.184(.079)$ \\
Length & .456 & .277 & $.51(.16)$ & $6.81(1)$ & $\mathbf{. 0 0 9}(.007)$ \\
Orange area & -.311 & .261 & $-13.24(5.13)$ & $4.50(1)$ & $\mathbf{. 0 3 4}(.008)$ \\
Black area & -.167 & .068 & $-7.28(8.17)$ & $.75(1)$ & $.385(.791)$ \\
Orange asymmetry & .228 & .091 & $.92(.66)$ & $2.36(1)$ & $.125(.432)$ \\
Black asymmetry & .571 & .434 & $1.63(.39)$ & $16.40(1)$ & $<.00001(.016)$ \\
Orange saturation & .372 & .184 & $3.00(1.18)$ & $5.10(1)$ & $\mathbf{. 0 2 4}(.046)$ \\
\hline
\end{tabular}

Note: We used a gamma error family with a log link function to test parameters explaining variation in our response variable: infection integral. We added 1 to all infection integrals to permit the use of the gamma distribution and log link function, which preliminary analyses indicated provided the best fit to the data. We reran the analysis excluding one extreme outlier infection integral of 447, and the same parameters were significant in both analyses (highlighted in boldface).
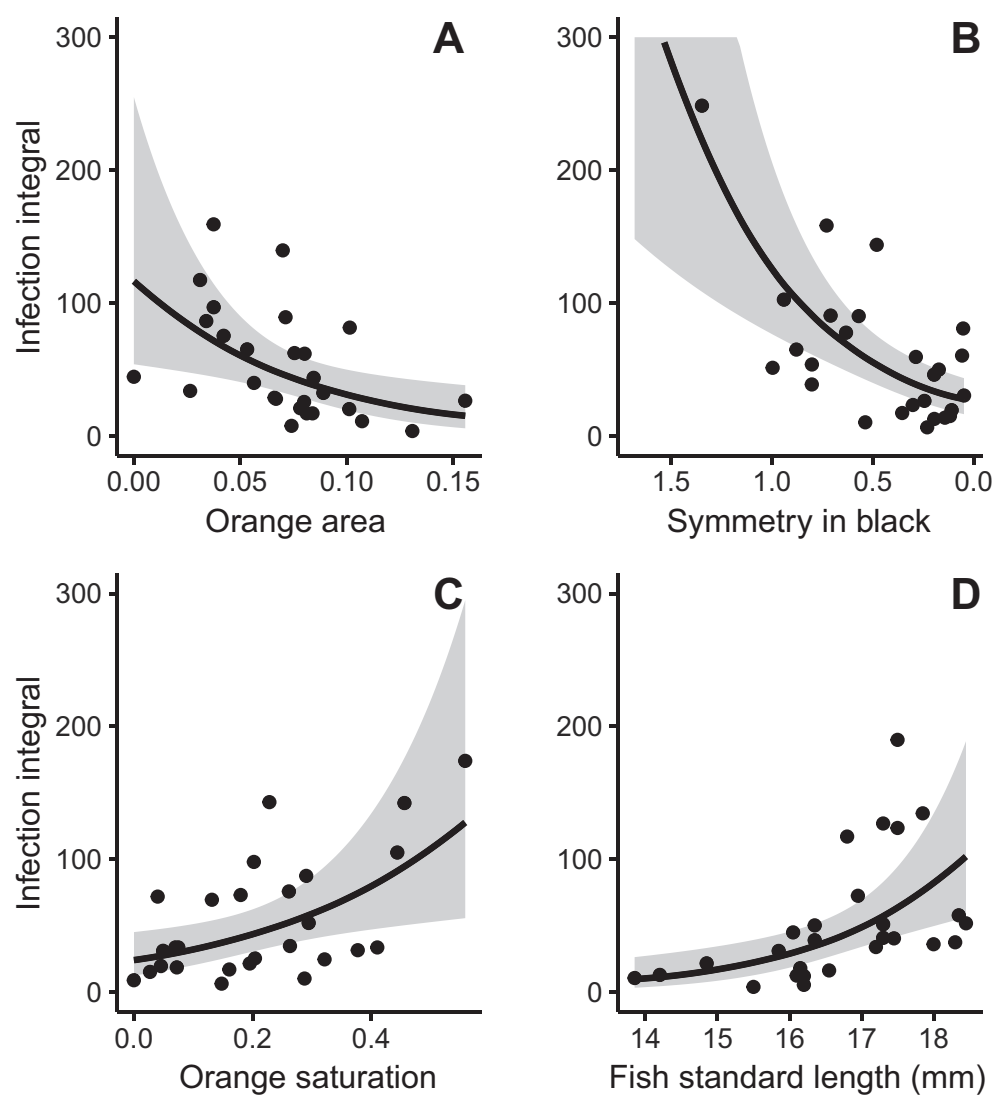

Figure 1: The size of a male's orange coloration $(A)$ and the left-right symmetry of his black coloration $(B)$, both static ornaments, were negatively correlated with his infection integral, or the area under the curve of number of Gyrodactylus turnbulli parasites through time $(A, B)$ : larger trait values predicted lower infection integrals and therefore higher parasite resistance. By contrast, the saturation of his orange coloration, a dynamic trait, was positively correlated with infection integral $(C)$ : larger trait values predicted higher infection integrals and therefore lower parasite resistance. Larger males $(D)$ developed higher infection integrals. Lines are the model fit, shading shows the $95 \%$ confidence intervals, and points indicate the partial residuals from the model in table 1, back transformed to the scale of the response variable and including the outlier. 

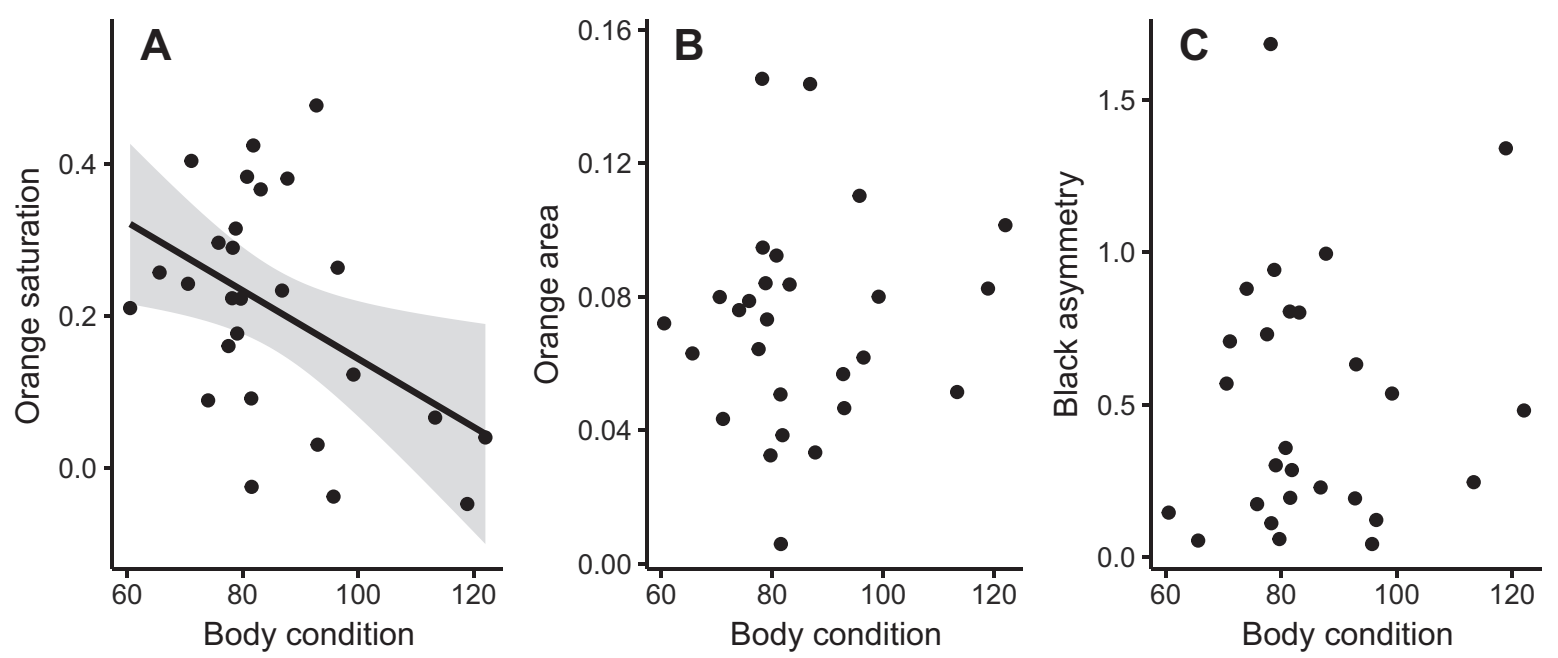

Figure 2: The dynamic $(A)$ but not the static $(B, C)$ male ornament was significantly negatively correlated with body condition. Lines are the model fit (only present in $A$ as this was the only significant result), shading shows the $95 \%$ confidence intervals, and points indicate the partial residuals from the models, back transformed to the scale of the response variable.

Our results support the hypothesis that symmetry in secondary sexual characteristics is indicative of male quality, in this case resistance to parasites. The ornaments of most males (at least 70\%) in our data set were more asymmetric than the female detection threshold suggested by Gross et al. (2007). If this level of asymmetry is representative of that among males in natural populations, females can use male ornament asymmetry as a reliable indicator of male quality. While Gross et al. (2007) do suggest that males can use behavior to mask asymmetry in orange, it is unclear whether asymmetry in orange is an important indicator; for example, we found no evidence that it indicates parasite resistance, in contrast to asymmetry in black. Additionally, no study has demonstrated female preference for symmetry in orange, whereas females do strongly prefer males with more symmetrical black ornaments (Sheridan and Pomiankowski 1997a), suggesting that males are unable to use behavior to mask this asymmetry. As the heritability of fluctuating asymmetry is generally low across systems, including ours (e.g., orange pigmentation in guppies [Gross et al. 2007], antlers in deer [Kruuk et al. 2003]), mating decisions based on fluctuating asymmetry probably do not provide indirect fitness benefits. They may instead provide direct fitness benefits to females if more symmetrical males are more resistant to parasites; such a correlation would help explain the evolution of female preference for symmetry. Indeed, these findings help explain the strong preference female guppies show for symmetrical males (Sheridan and Pomiankowski 1997a); symmetry is correlated with parasite resistance, despite not being correlated with overall genetic quality (Sheridan and Pomiankowski 1997b).
The relationship between guppy carotenoid coloration and parasite resistance is complex. We found that males with larger areas of carotenoid coloration, a static trait, are more resistant to parasites. Males from other guppy populations with larger areas of orange also demonstrate superiority in other traits, such as swimming speeds, display rates, and sperm quality (e.g., Nicoletto 1993; Pitcher et al. 2007). Females from many populations, including our focal population, prefer these males (Houde and Endler 1990; Evans et al. 2003, 2004; Devigili et al. 2015), and in natural conditions they sire more offspring (Gordon et al. 2015). As males with larger areas of orange suffer higher predation (Godin and McDonough 2003) and parasitism further increases this risk (Stephenson et al. 2016), predation may ensure the honesty of this signal.

We found that males with more saturated carotenoid coloration, a condition-dependent dynamic trait (KodricBrown 1989; Grether 2000), were less resistant to infection. Our males may have preferentially allocated carotenoids to saturating skin pigments (and hence attracting females; Grether 2000) rather than immune function. Consistent with the idea that males may trade off investment in this dynamic ornament with other resource-dependent traits, we found that orange saturation (but neither of the static predictive ornaments) was significantly negatively correlated with male body condition (fig. 2). Sexual signaling theory predicts that while females attempt to accurately assess male quality during mate choice, males attempt to "game" the system (Johnstone 2000; Gross et al. 2007). Elements of this system may be conducive to male deceit: Gyrodactylus spp. parasitism is highly spatially and temporally 
variable and is aggregated within host populations (Stephenson et al. 2015b), so the per capita infection risk is often low. Furthermore, due to sperm storage, males can sire offspring even 10 months posthumously (Lopez-Sepulcre et al. 2013). Under these conditions, biasing carotenoid investment toward mate attraction to the extent of handicapping the immune system may be successful often enough to have evolved as a male strategy. However, whether investment of carotenoids in ornaments is actually a handicap is not well resolved (Koch et al. 2018; Weaver et al. 2018). Additionally, carotenoids in the skin may be mobilized by the immune system to combat infections once they occur (Lozano 1994). This difference in reliability between the area and saturation of carotenoid signals explains why females, at least from one population, prioritize area over saturation in their mating preferences (Karino et al. 2010). Alternatively, males with more saturated orange spots may be higher-quality hosts for the parasite (Seppälä et al. 2008), or high levels of carotenoid may benefit the parasite more than the host (Hõrak et al. 2004). This result is consistent with previous work across systems; the underlying proximate and ultimate mechanisms warrant further investigation (Hõrak et al. 2004; Kolluru et al. 2006).

Overall, our results suggest that more attractive males are more resistant to infection, but field surveys have failed to find a correlation between male attractiveness and parasitism (Martin and Johnsen 2007; Gotanda et al. 2013). This apparently contradictory result, along with similar previous results (Møller et al. 1999), may have an epidemiological explanation. Our laboratory-controlled infection exposed all males to the same infection risk, but more attractive males may have more contacts with potentially infected individuals ( $\beta_{c}$; Hawley et al. 2011), as a result of increased mating opportunities (e.g., Houde and Torio 1992). Our findings suggest that more attractive males are more resistant and may therefore be less heavily infected and thus less infectious $\left(\beta_{\mathrm{p}}\right.$; Hawley et al. 2011; Stephenson et al. 2017). Given that the transmission rate is $\beta_{\mathrm{p}} \times \beta_{\mathrm{c}}$ (Hawley et al. 2011), these two terms may have equal weight in determining transmission: the increase in $\beta_{c}$ due to increased attractiveness is counterbalanced by the decrease in $\beta_{\mathrm{p}}$ due to reduced infectiousness, resulting in the lack of correlation observed in field surveys. How infection avoidance behavior (Stephenson et al. 2016, 2018) or parasite-mediated behavioral plasticity in these males (Stephenson 2019) might contribute to these dynamics remains an open question.

In this well-characterized system, we can explain the maintenance of heritable genetic variation in the face of strong selection without invoking the host-parasite coadaptational cycles underlying Hamilton and Zuk's (1982) hypothesis (Lozano 1994). We show that the traits females prefer are honest indicators of male resistance to parasites, yet substantial genetic variation in male guppy color patterns is maintained (Hughes et al. 2005). The multifarious selection pressures acting on these traits may resolve this apparent paradox (Maynard Smith 1978). Sexual selection (and parasite-mediated natural selection; present study) for larger areas of carotenoid coloration is counteracted by natural selection by predators for less conspicuous coloration (Endler 1980). Male color patterns also evolve rapidly in response to changes in environmental conditions, such as light intensity (Endler 1983), dietary carotenoid availability (Grether et al. 2001), and predation regime (Endler 1983; Kemp et al. 2009). Furthermore, color pattern novelty confers higher reproductive fitness (Hughes et al. 2013) and some defense against predators (Olendorf et al. 2006) in natural conditions. We suggest that the spatial and temporal variation in pressure from Gyrodactylus parasites across natural guppy populations (Martin and Johnsen 2007; Gotanda et al. 2013; Stephenson et al. 2015b) may contribute to the observed and unexplained population-level variation in male coloration (Millar et al. 2006) and female preferences (Houde and Endler 1990).

In conclusion, our results show that male secondary sexual characteristics can honestly indicate resistance to parasites, even in the absence of infection. We also demonstrate that ornaments spanning the continuum from static to dynamic are informative of a male's relationship with his parasites. Combined, these findings indicate that the role of parasites in sexual selection is likely broader than that encompassed by previous hypotheses.

\section{Acknowledgments}

We thank J. Cable, C. Kinsella, M. Reynolds, K. Simmler, S. Smithers, and C. van Oosterhout for technical assistance and useful discussions. K. A. Young made helpful comments on the manuscript. This work was funded by the Fisheries Society of the British Isles (FSBI; PhD studentship) and the Center for Adaptation to a Changing Environment (ACE; postdoctoral fellowship).

\section{Statement of Authorship}

J.F.S. designed the study and collected the data with substantial assistance from M.S., J.J., and J.T., using the software developed by J.T. J.F.S. analyzed the data with input from J.J. J.F.S. wrote and revised the manuscript, incorporating comments from all authors.

\section{Literature Cited}

Able, D. J. 1996. The contagion indicator hypothesis for parasitemediated sexual selection. Proceedings of the National Academy of Sciences of the USA 93:2229-2233. 
Adelman, J. S., L. Kirkpatrick, J. L. Grodio, and D. M. Hawley. 2013. House finch populations differ in early inflammatory signaling and pathogen tolerance at the peak of Mycoplasma gallisepticum infection. American Naturalist 181:674-689.

Agnew, P., and J. C. Koella. 1997. Virulence, parasite mode of transmission and host fluctuating asymmetry. Proceedings of the Royal Society B 264:9-15.

Allenbach, D. M., K. B. Sullivan, and M. J. Lydy. 1999. Higher fluctuating asymmetry as a measure of susceptibility to pesticides in fishes. Environmental Toxicology and Chemistry 18:899-905.

Andersson, M. 1994. Sexual selection. Princeton University Press, Princeton, NJ.

Blount, J. D., N. Metcalfe, T. R. Birkhead, and P. F. Surai. 2003. Carotenoid modulation of immune function and sexual attractiveness in zebra finches. Science 300:125-127.

Borgia, G. 1986. Satin bowerbird parasites: a test of the bright male hypothesis. Behavioral Ecology and Sociobiology 19:355-358.

Dargent, F., M. E. Scott, A. P. Hendry, and G. F. Fussman. 2013. Experimental elimination of parasites in nature leads to the evolution of increased resistance in hosts. Proceedings of the Royal Society B 280:20132371.

Dawson, R. D., and G. R. Bortolotti. 2006. Carotenoid-dependent coloration of male American kestrels predicts ability to reduce parasitic infections. Naturwissenschaften 93:597-602.

Devigili, A., J. P. Evans, A. Di Nisio, and A. Pilastro. 2015. Multivariate selection drives concordant patterns of pre- and postcopulatory sexual selection in a livebearing fish. Nature Communications 6:8291.

Endler, J. A. 1978. A predator's view of animal color patterns. Evolutionary Biology 11:319-364.

- 1980. Natural selection on color patterns in Poecilia reticulata. Evolution 34:76-91.

- 1983. Natural and sexual selection on colour patterns in poecilid fishes. Environmental Biology of Fishes 9:173-190.

Evans, J. P., A. Bisazza, and A. Pilastro. 2004. Female mating preferences for colourful males in a population of guppies subject to high predation. Journal of Fish Biology 65:1154-1159.

Evans, J. P., T. E. Pitcher, and A. E. Magurran. 2002. The ontogeny of courtship, colour and sperm production in male guppies. Journal of Fish Biology 60:495-498.

Evans, J. P., L. Zane, S. Francescato, and A. Pilastro. 2003. Directional postcopulatory sexual selection revealed by artificial insemination. Nature 421:360-363.

Faivre, B., A. Grégoire, M. Préault, F. Cézilly, and G. Sorci. 2003. Immune activation rapidly mirrored in a secondary sexual trait. Science 300:103.

Folstad, I., and A. J. Karter. 1992. Parasites, bright males, and the immunocompetence handicap. American Naturalist 139:603622.

Forstmeier, W., and H. Schielzeth. 2010. Cryptic multiple hypothesis testing in linear models: overestimated effect sizes and the winner's curse. Behavioral Ecology and Sociobiology 65:47-55.

Gilbert, R., and G. W. Uetz. 2016. Courtship and male ornaments as honest indicators of immune function. Animal Behaviour 117:97-103.

Godin, J. G. J., and H. E. McDonough. 2003. Predator preference for brightly colored males in the guppy: a viability cost for a sexually selected trait. Behavioral Ecology 14:194-200.

Goodwin, T. W. 1986. Metabolism, nutrition, and function of carotenoids. Annual Review of Nutrition 6:271-297.
Gordon, S. P., D. N. Reznick, J. D. Arendt, A. Roughton, M. N. Ontiveros Hernandez, P. Bentzen, and A. Lopez-Sepulcre. 2015. Selection analysis on the rapid evolution of a secondary sexual trait. Proceedings of the Royal Society B 282:20151244.

Gotanda, K. M., L. C. Delaire, J. A. M. Raeymaekers, F. PérezJvostov, F. Dargent, P. Bentzen, M. E. Scott, et al. 2013. Adding parasites to the guppy-predation story: insights from field surveys. Oecologia 172:155-166.

Grether, G. F. 2000. Carotenoid limitation and mate preference evolution: a test of the indicator hypothesis in guppies (Poecilia reticulata). Evolution 54:1712-1724.

Grether, G. F., M. E. Cummings, and J. Hudon. 2005. Countergradient variation in the sexual coloration of guppies (Poecilia reticulata): drospterin synthesis balances carotenoid availability. Evolution 59:175-188.

Grether, G. F., J. Hudon, and J. A. Endler. 2001. Carotenoid scarcity, synthetic pteridine pigments and the evolution of sexual coloration in guppies. Proceedings of the Royal Society B 268:1245-1253.

Grether, G. F., S. Kasahara, G. R. Kolluru, and E. L. Cooper. $2004 a$. Sex-specific effects of carotenoid intake on the immunological response to allografts in guppies (Poecilia reticulata). Proceedings of the Royal Society B 271:45-49.

Grether, G. F., G. R. Kolluru, and K. Nersissian. 2004b. Individual colour patches and multicomponent signals. Biological Reviews 79:583-610.

Grether, G. F., G. R. Kolluru, K. Lin, M. A. Quiroz, G. Robertson, and A. J. Snyder. 2008. Maternal effects of carotenoid consumption in guppies (Poecilia reticulata). Functional Ecology 22:294-302.

Gross, M. R., H. Y. Suk, and C. T. Robertson. 2007. Courtship and genetic quality: asymmetric males show their best side. Proceedings of the Royal Society B 274:2115-2122.

Hamilton, W. D., and M. Zuk. 1982. Heritable true fitness and bright birds: a role for parasites? Science 218:384-387.

Hamilton, W. J., and R. Poulin. 1997. The Hamilton and Zuk hypothesis revisited: a meta-analytical approach. Behaviour 134:299-320.

Hawley, D. M., R. S. Etienne, V. O. Ezenwa, and A. E. Jolles. 2011. Does animal behavior underlie covariation between hosts' exposure to infectious agents and susceptibility to infection? implications for disease dynamics. Integrative and Comparative Biology $51: 528-539$.

Hill, G. E., and K. L. Farmer. 2005. Carotenoid-based plumage coloration predicts resistance to a novel parasite in the house finch. Naturwissenschaften 92:30-34.

Hill, J. A., D. A. Enstrom, E. D. Ketterson, V. Nolan Jr., and C. Ziegenfus. 1999. Mate choice based on static versus dynamic secondary sexual traits in the dark-eyed junco. Behavioral Ecology 10:91-96.

Hõrak, P., P. F. Surai, I. Ots, and A. P. Møller. 2004. Fat soluble antioxidants in brood-rearing great tits Parus major: relations to health and appearance. Journal of Avian Biology 35:63-70.

Houde, A. E. 1997. Sex, color and mate choice in guppies. Monographs in Behavior and Ecology. Princeton University Press, Princeton, NJ.

Houde, A. E., and J. A. Endler. 1990. Correlated evolution of female mating preferences and male color patterns in the guppy Poecilia reticulata. Science 248:1405-1408.

Houde, A. E., and A. J. Torio. 1992. Effect of parasitic infection on male color pattern and female choice in guppies. Behavioral Ecology 3:346-351. 
Hughes, K. A., A. E. Houde, A. C. Price, and F. H. Rodd. 2013. Mating advantage for rare males in wild guppy populations. $\mathrm{Na}-$ ture 503:108-110.

Hughes, K. A., F. H. Rodd, and D. N. Reznick. 2005. Genetic and environmental effects on secondary sex traits in guppies (Poecilia reticulata). Journal of Evolutionary Biology 18:35-45.

Johnstone, R. A. 2000. Game theory and communication. Pages 94117 in L. A. Dugatkin and H. K. Reeve, eds. Game theory and animal behavior. Oxford University Press, New York.

Karino, K., and Y. Hajima. 2004. Algal-diet enhances sexual ornament, growth, and reproduction in the guppy. Behaviour 141: 585-601.

Karino, K., Y. Shimada, and A. Sato. 2010. Relative importance of the area and intensity of the orange spots of male guppies Poecilia reticulata as mating traits preferred by females. Journal of Fish Biology 77:299-307.

Kemp, D. J., F.-K. Batista, and D. N. Reznick. 2018. Predictable adaptive trajectories of sexual coloration in the wild: evidence from replicate experimental guppy populations. Evolution 72: 2462-2477.

Kemp, D. J., D. N. Reznick, G. F. Grether, and J. A. Endler. 2009. Predicting the direction of ornament evolution in Trinidadian guppies (Poecilia reticulata). Proceedings of the Royal Society B 276:4335-4343.

Koch, R. E., A. N. Kavazis, D. Hasselquist, W. R. Hood, Y. Zhang, M. B. Toomey, and G. E. Hill. 2018. No evidence that carotenoid pigments boost either immune or antioxidant defenses in a songbird. Nature Communications 9:491.

Kodric-Brown, A. 1989. Dietary carotenoids and male mating success in the guppy: an environmental component to female choice. Behavioral Ecology and Sociobiology 25:393-401.

Kodric-Brown, A., and J. H. Brown. 1984. Truth in advertising: the kinds of traits favored by sexual selection. American Naturalist 124:309-323.

Kolluru, G. R., G. F. Grether, S. H. South, E. Dunlop, A. Cardinali, L. Liu, and A. Carapiet. 2006. The effects of carotenoid and food availability on resistance to a naturally occurring parasite (Gyrodactylus turnbulli) in guppies (Poecilia reticulata). Biological Journal of the Linnean Society 89:301-309.

Kruuk, L. E. B., J. Slate, J. M. Pemberton, and T. Clutton-Brock. 2003. Fluctuating asymmetry in a secondary sexual trait: no associations with individual fitness, environmental stress or inbreeding, and no heritability. Journal of Evolutionary Biology 16:101-113.

Lens, L., S. van Dongen, S. Kark, and E. Matthysen. 2002. Fluctuating asymmetry as an indicator of fitness: can we bridge the gap between studies? Biological Reviews 77:27-38.

Lindström, K., and J. Lundström. 2000. Male greenfinches (Carduelis chloris) with brighter ornaments have higher virus infection clearance rate. Behavioral Ecology and Sociobiology 48:44-51.

Loehle, C. 1995. Social barriers to pathogen transmission in wild animal populations. Ecology 76:326-335.

Lopez-Sepulcre, A., S. P. Gordon, I. G. Paterson, P. Bentzen, and D. N. Reznick. 2013. Beyond lifetime reproductive success: the posthumous reproductive dynamics of male Trinidadian guppies. Proceedings of the Royal Society B 280:20131116.

Lozano, G. A. 1994. Carotenoids, parasites, and sexual selection. Oikos 70:309-311

Martin, C. H., and S. Johnsen. 2007. A field test of the HamiltonZuk hypothesis in the Trinidadian guppy (Poecilia reticulata). Behavioral Ecology and Sociobiology 61:1897-1909.
Maynard Smith, J. 1978. The evolution of sex. Cambridge University Press, London.

Meadows, M. G., N. I. Morehouse, R. L. Rutowski, J. M. Douglas, and K. J. McGraw. 2011. Quantifying iridescent coloration in animals: a method for improving repeatability. Behavioral Ecology and Sociobiology 65:1317-1327.

Milinski, M., and T. C. M. Bakker. 1990. Female sticklebacks use male coloration in mate choice and hence avoid parasitized males. Nature 344:330-333.

Millar, N. P., D. N. Reznick, M. T. Kinnison, and A. P. Hendry. 2006. Disentangling the selective factors that act on male colour in wild guppies. Oikos 113:1-12.

Møller, A. P., P. Christe, and E. Lux. 1999. Parasitism, host immune function, and sexual selection. Quarterly Review of Biology $74: 3-20$.

Nicoletto, P. 1993. Female sexual response to condition-dependent ornaments in the guppy, Poecilia reticulata. Animal Behaviour 46:441-450.

Olendorf, R., F. H. Rodd, D. Punzalan, A. E. Houde, C. Hunt, D. N. Reznick, and K. A. Hughes. 2006. Frequency-dependent survival in natural guppy populations. Nature 441:633-636.

Palmer, A. R., and C. Strobeck. 1986. Fluctuating asymmetry: measurement, analysis, patterns. Annual Review of Ecology and Systematics 17:391-421.

Peig, J., and A. J. Green. 2009. New perspectives for estimating body condition from mass/length data: the scaled mass index as an alternative method. Oikos 118:1883-1891.

Phillips, K. P., J. Cable, R. S. Mohammed, M. Herdegen-Radwan, J. Raubic, K. J. Przesmycka, C. van Oosterhout, et al. 2018. Immunogenetic novelty confers a selective advantage in hostpathogen coevolution. Proceedings of the National Academy of Sciences of the USA 115:1552-1557.

Pitcher, T. E., F. H. Rodd, and L. Rowe. 2007. Sexual colouration and sperm traits in guppies. Journal of Fish Biology 70:165-177.

Poulin, R., and W. L. Vickery. 1993. Parasite distribution and virulence: implications for parasite-mediated sexual selection. Behavioral Ecology and Sociobiology 33:429-436.

R Core Team. 2018. R: a language and environment for statistical computing. R Foundation for Statistical Computing, Vienna.

Schneider, C. A., W. S. Rasband, and K. W. Eliceiri. 2012. NIH Image to ImageJ: 25 years of image analysis. Nature Methods 9:671675.

Seppälä, O., K. Liljeroos, A. Karvonen, and J. Jokela. 2008. Host condition as a constraint for parasite reproduction. Oikos 117: 749-753.

Shaw, D. J., and A. P. Dobson. 1995. Patterns of macroparasite abundance and aggregation in wildlife populations: a quantitative review. Parasitology 111:111-133.

Sheridan, L., and A. Pomiankowski. 1997a. Female choice for spot asymmetry in the Trinidadian guppy. Animal Behaviour 54: $1523-1530$.

- 1997b. Fluctuating asymmetry, spot asymmetry and inbreeding depression in the sexual coloration of male guppy fish. Heredity 79:515-523.

Siva-Jothy, M. T. 2000. A mechanistic link between parasite resistance and expression of a sexually selected trait in a damselfly. Proceedings of the Royal Society B 267:2523-2527.

Stephenson, J. F. 2019. Parasite-induced plasticity in host social behaviour depends on sex and susceptibility. Biology Letters 15:20190557. 
Stephenson, J. F., C. Kinsella, J. Cable, and C. van Oosterhout. 2016. A further cost for the sicker sex? evidence for male-biased parasite-induced vulnerability to predation. Ecology and Evolution 6:2506-2515.

Stephenson, J. F., S. E. Perkins, and J. Cable. 2018. Transmission risk predicts avoidance of infectious conspecifics in Trinidadian guppies. Journal of Animal Ecology 87:1525-1533.

Stephenson, J. F., and M. Reynolds. 2016. Imprinting can cause a maladaptive preference for infectious conspecifics. Biology Letters 12:20160020.

Stephenson, J. F., M. Stevens, J. Troscianko, and J. Jokela. 2020. Data from: The size, symmetry, and color saturation of a male guppy's ornaments forecast his resistance to parasites. American Naturalist, Dryad Digital Repository, https://doi.org/10.5061/dryad $.547 \mathrm{~d} 7 \mathrm{wm} 61$.

Stephenson, J. F., C. van Oosterhout, and J. Cable. 2015a. Pace of life, predators and parasites: predator-induced life history evolution in Trinidadian guppies predicts decrease in parasite tolerance. Biology Letters 11:20150806.

Stephenson, J. F., C. van Oosterhout, R. S. Mohammed, and J. Cable. 2015b. Parasites of Trinidadian guppies: evidence for sexand age-specific trait-mediated indirect effects of predators. Ecology 96:489-498.

Stephenson, J. F., K. A. Young, J. Fox, J. Jokela, J. Cable, and S. E. Perkins. 2017. Host heterogeneity affects both parasite transmission to and fitness on subsequent hosts. Philosophical Transactions of the Royal Society B 372:20160093.

Stevens, M., C. A. Párraga, I. Cuthill, J. C. Partridge, and T. S. Troscianko. 2007. Using digital photography to study animal coloration. Biological Journal of the Linnean Society 90:211-237.

Stevens, M., M. C. Stoddard, and J. P. Higham. 2009. Studying primate color: towards visual system dependent methods. International Journal of Primatology 30:893-917.
Stoffel, M. A., S. Nakagawa, and H. Schielzeth. 2017. rptR: repeatability estimation and variance decomposition by generalized linear mixed-effects models. Methods in Ecology and Evolution 8:1639-1644

Swaddle, J. P., M. S. Witter, and I. C. Cuthill. 1994. The analysis of fluctuating asymmetry. Animal Behaviour 48:986-989.

Troscianko, J., and M. Stevens. 2015. Image calibration and analysis toolbox - a free software suite for objectively measuring reflectance, colour and pattern. Methods in Ecology and Evolution 6:1320-1331.

van Oosterhout, C., R. S. Mohammed, H. Hansen, G. A. Archard, M. McMullan, D. J. Weese, and J. Cable. 2007. Selection by parasites in spate conditions in wild Trinidadian guppies (Poecilia reticulata). International Journal for Parasitology 37:805812.

van Oosterhout, C., R. E. Trigg, G. R. Carvalho, A. E. Magurran, L. Hauser, and P. W. Shaw. 2003. Inbreeding depression and genetic load of sexually selected traits: how the guppy lost its spots. Journal of Evolutionary Biology 16:273-281.

Weaver, R. J., E. S. A. Santos, A. M. Tucker, A. E. Wilson, and G. E. Hill. 2018. Carotenoid metabolism strengthens the link between feather coloration and individual quality. Nature Communications 9:73.

White, E. M., J. C. Partridge, and S. C. Church. 2003. Ultraviolet dermal reflexion and mate choice in the guppy, Poecilia reticulata. Animal Behaviour 65:693-700.

Wingfield, J. C., R. E. Hegner, A. M. Dufty Jr., and G. F. Ball. 1990. The "challenge hypothesis": theoretical implications for patterns of testosterone secretion, mating systems, and breeding strategies. American Naturalist 136:829-846.

Associate Editor: David N. Reznick Editor: Russell Bonduriansky

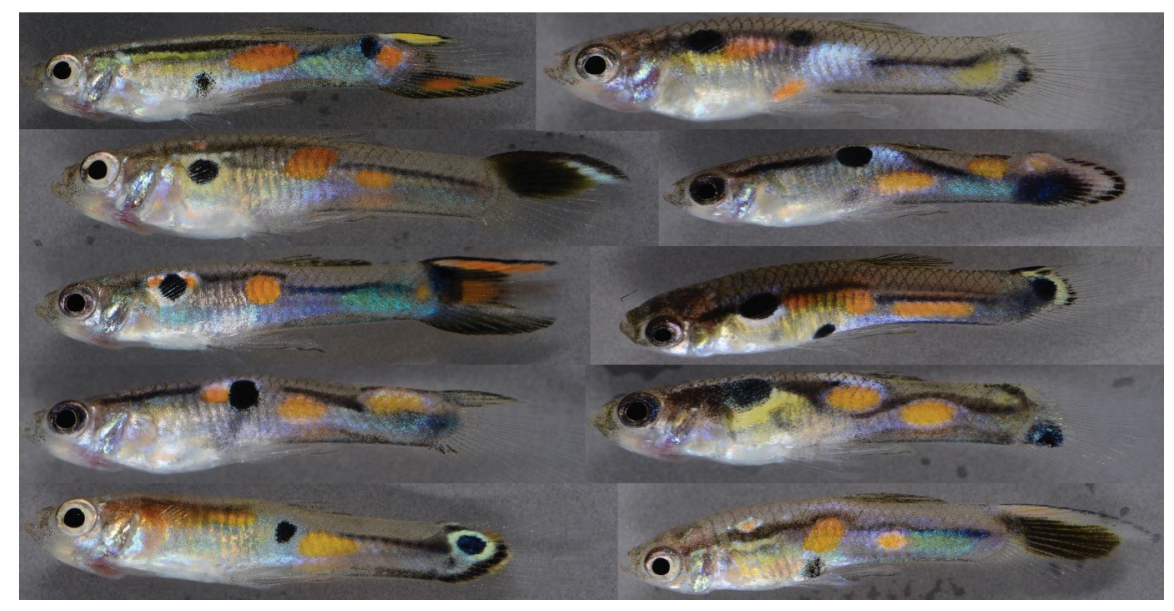

Male guppies (Poecilia reticulata) have highly variable color patterns that indicate their resistance to parasites, even before they become infected. Photo credit: Jessica Stephenson. 